\title{
Digital processing of Cherenkov light signals from extensive air showers of cosmic rays
}

\author{
A.A. Ivanov* \\ Shafer Institute for Cosmophysical Research \& Aeronomy \\ E-mail: ivanoveikfia.ysn.ru
}

\section{S.V. Matarkin}

Shafer Institute for Cosmophysical Research \& Aeronomy

E-mail: matarkinsveikfia.ysn.ru

\section{L.V. Timofeev}

Shafer Institute for Cosmophysical Research \& Aeronomy

E-mail: timofeevlev@ikfia.ysn.ru

\begin{abstract}
The operation of a wide field-of-view Cherenkov telescope is described. The detection of extensive air showers (EAS) of cosmic rays (CR) is based upon the coincidence with signals from the Yakutsk array. The data acquisition system of the telescope yields signals connected with EAS development parameters: presumably, shower age and position of shower maximum in the atmosphere. Here we describe the application of digital signal processing in order to reconstruct Cherenkov radiation signals induced by CR showers.
\end{abstract}

36th International Cosmic Ray Conference -ICRC2019-

July 24th - August 1st, 2019

Madison, WI, U.S.A.

${ }^{*}$ Speaker. 


\section{Introduction}

Ultra-high energy cosmic rays (UHECR) entering Earth's atmosphere create a cascade of secondary particles. In this cascade there are myriad of charged particles moving at speeds greater than $c / n$, where $c$ is the velocity of light in vacuum and $n$ is the index of refraction in air. These particles emit coherent Cherenkov radiation [1] that contains information about the energy, composition and arrival direction of the primary particle that initiated the extensive air shower (EAS) [2].

Since the first observation by Galbraith and Jelley [3] and a systematic measurement of air Cherenkov radiation properties in the Pamir experiment [4], a number of EAS arrays have been equipped with Cherenkov radiation detectors. Particularly, in the Yakutsk array experiment these detectors are used to estimate the energy and mass composition of the primaries [5, 6].

In a majority of previous measurements, analog signal readout systems were used that had narrow bandwidth, which restricted the possibility of pulse-shape reconstruction of the Cherenkov radiation from EAS; or, detectors were designed for measurement of the integral signal $[5,7,8]$. In this paper, we describe a method for reconstructing the pulse shape of the Cherenkov radiation from EAS as detected using a wide field-of-view Cherenkov telescope (hereinafter 'telescope') based on the coincidence of signals with the Yakutsk array.

\section{A Cherenkov telescope of the Yakutsk array}

The constituent parts of the telescope are a) the spherical mirror ( $\varnothing 260 \mathrm{~mm}, f=113 \mathrm{~mm}$ ) mounted at the bottom of a metal tube; b) a position-sensitive photomultiplier tube (PMT; Hamamatsu R2486; $\varnothing 50 \mathrm{~mm}$ ) at the focus for which the anode is formed by $16 \times 16$ crossed wires; c) a voltage-divider circuit and mechanical support attached to the bearing plate; and d) 32 operational amplifiers mounted onto the tube. The telescope is mounted vertically near an array station. A comprehensive description of telescope can be found in Refs. [9, 10, 11].

The data acquisition system (DAS) of the telescope consists of 32 operational amplifiers that have 300-MHz bandwidth AD8055 chips connected by long $(12 \mathrm{~m})$ coaxial cables to 8-bit LAn4USB ADC digitizers with 4-ns time slicing. All of the ADC output signals from the 32 channels are continuously stored in PC memory. A trigger signal from the EAS array terminates the process and signals in a 16/32 $\mu$ s interval preceding a trigger are dumped. In Fig. 1, an example is given of the output signals of the DAS recorded in coincidence with the Yakutsk array detectors in a particular CR shower. The EAS parameters are estimated using the data from the surface array detectors. Twelve wires (in arbitrary order) exhibit Cherenkov radiation signals; the other twenty wires do not show a significant signal above the noise level.

In this paper, we describe an analysis of data that were accumulated during the period 20122016 for which EAS events were detected simultaneously by the surface detectors and the telescope. Data selection cuts are applied to exclude showers with cores out of the array area and with zenith angles $\theta>60^{\circ}$. In the present analysis, we are not using the angular dependence of the telescope signals in an individual EAS event; the angular and arrival time differences of signals are ignored. 

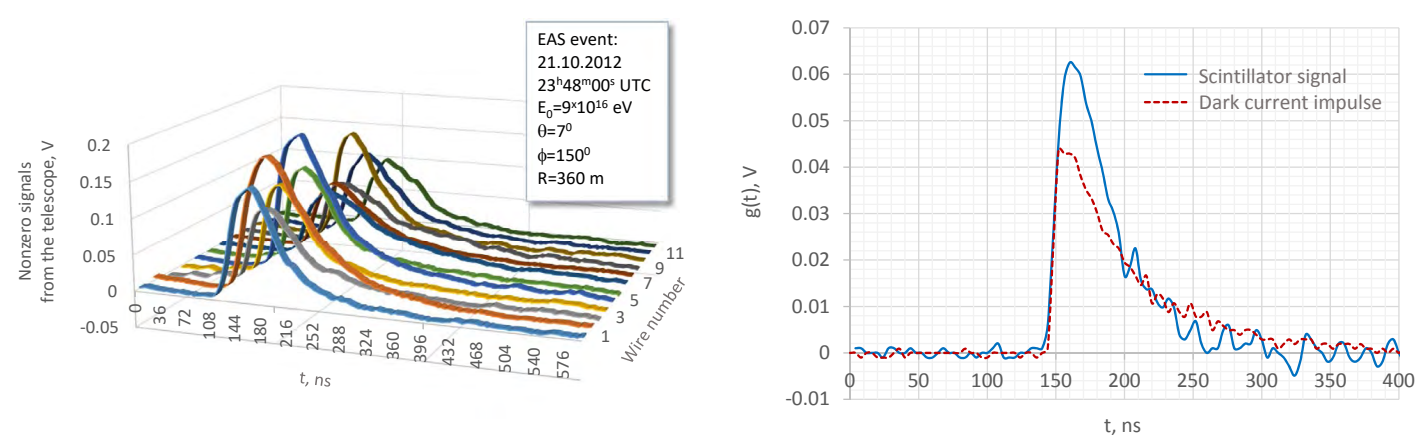

Figure 1: Left panel: Output signals of WFOV Cherenkov telescope's data acquisition system from EAS event. Right panel: Impulse response of the system to short input signals.

\section{Deconvolution of Cherenkov telescope signals}

All of the components of the telescope's DAS - amplifiers, ADCs, etc.- are linear devices; the output signal can be represented as a convolution of the input signal $f_{\text {in }}(t)$ with a system transfer function $g(t)$

$$
f_{\text {out }}(t)=\int_{-\infty}^{\infty} f_{\text {in }}(\tau) g(t-\tau) d \tau=\left(f_{\text {in }} * g\right) .
$$

Applying a delta function as input signal, the convolution represents the impulse response of the system. Here, the impulse is a signal composed of all zeros except for a single nonzero point: a digital equivalent of the delta function.

A straightforward way for reconstructing the input signal is a deconvolution using the Fourier transform of signals

$$
\hat{f}(v)=\int_{-\infty}^{\infty} e^{-2 \pi i v t} f(t) d t .
$$

In the frequency domain, the convolution relation is $\hat{f_{\text {out }}}=\hat{f_{\text {in }}} \times \hat{g}$ and the deconvolution is given by $\hat{f_{\text {in }}}=\hat{f_{\text {out }}} / \hat{g}$, where hats symbolize Fourier transforms [12].

\subsection{Impulse response of the data acquisition system}

The impulse response of the DAS - its output when presented with a brief input signal - is ultimately used as a function that is deconvolved from operational signals in order to obtain unknown input signals. We have tested the DAS with two different signal sources that are brief enough in duration to be considered as good approximations to a delta function: the plastic scintillator and the dark current impulse of the PMT.

In the first case we have used the plastic scintillator common for the Yakutsk array detectors consisting of a piece of polymethyl methacrylate with dissolved n-terphenyl (PPP) and 1,4-di-5phenyl-2-oxazolyl-benzene (POPOP) scintillators. The decay time constant of scintillation is 2.7 ns [13].

The second source tested is the dark current impulse of the shielded PMT produced by an electron beam emitted upon overvoltage. It is important to maintain the anode voltage at a minimal excess of its voltage limit in order to generate a single impulse rather than a multitude of impulses 

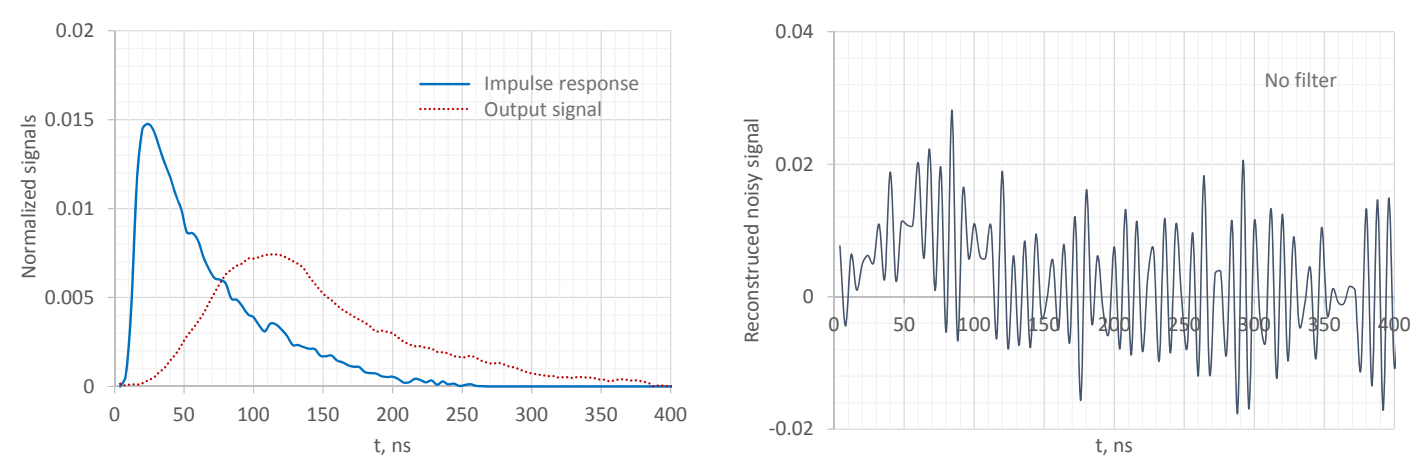

Figure 2: An example of reconstruction of the noisy signal. Left panel: Impulse response and the measured output signal of the system; Right panel: deconvolved input signal.

forming the current. The pulse shape is determined by the particular PMT time response. Our Hamamatsu R2486 position sensitive PMT has a rise time 5.5 ns.

In Fig. 1, the two resulting responses from our two sources are shown. Indeed, the durations of the output signals in our system are much longer than those of input signals used. Consequently, both sources can be considered as suitable substitute for the input of a delta function. For the analysis presented here, we have used the impulse response to dark current impulses averaged over 18 measurements made 14 March 2018 (Fig. 2, left panel); this choice admits of no need for additional experimental equipment.

\subsection{Noise reduction}

Having measured the impulse response, we may deconvolve input signals that have durations greater than the duration of $g(t)$. In practice, noise of natural and instrumental origin is added to signals. In Fig. 2, an illustration is given of a real, noisy input signal naively deconvolved using the impulse response presented in the previous section. In order to carry out the Fourier (and inverse) transforms of digital signals, the fast Fourier transform (FFT) algorithm is used [14]. In the present analysis, we disregard inter-signal time differences in the data from telescope, shifting signals to the same starting point. The tails of the signals are truncated by an appropriate time window.

From the results shown in Fig. 2, it is evident that there is a need to use a noise reduction filter for the data from the telescope. We have chosen from the variety of available filters in the field the Wiener filter [15] working in the frequency domain, characterized by the minimum impact of deconvolved noise at frequencies that have a poor signal-to-noise ratio (SNR).

\subsubsection{Wiener deconvolution algorithm}

The goal of the approach in this section is to find some function $w(t)$ so that the transform of the input signal can be evaluated as $\hat{f_{\text {in }}}=\hat{w} \hat{\text { out }}$, where an estimate of $f_{\text {in }}$ minimizes the mean square error. Wiener showed that the filter

$$
\hat{w}=\frac{1}{\hat{g}}\left[\frac{|\hat{g}|^{2}}{|\hat{g}|^{2}+\frac{N(f)}{S(f)}}\right]
$$




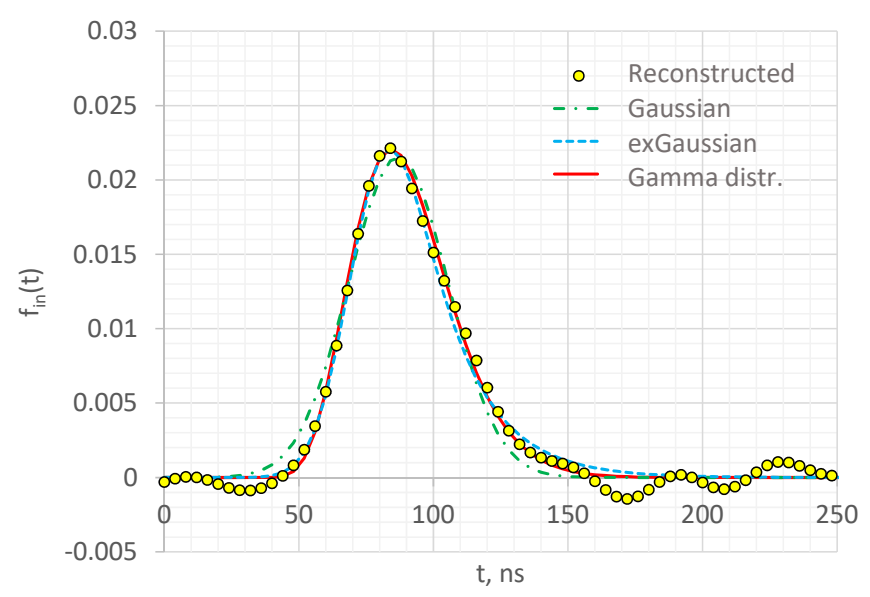

Figure 3: Analytic approximations to the reconstructed input Cherenkov signal from EAS.

provides such a function in the frequency domain, where $N(f)$ and $S(f)$ are the mean power spectral density of the noise and input signal, respectively [15]. When the noise is zero, the expression reduces to $\hat{f_{\text {in }}}=\hat{f_{\text {out }}} / \hat{\mathrm{g}}$. The ratio $S(f) / N(f)$ is the representation of the SNR. Therefore, the Wiener filter attenuates frequencies depending on the signal-to-noise ratio.

The minimum time window of the DAS of the telescope is $16 \mu \mathrm{s}$, while the maximum duration of the Cherenkov signal is $300 \mathrm{~ns}$, so we have at least 50 time windows where only the noise is detected from which $N(f)$ can be estimated. On the other hand, we do not know the power spectral density of the input signal. The only possibility is to estimate it using the output signal.

In Fig. 3 a sample of the resulting Wiener deconvolution of signals of EAS event detected on 21.10.2012 (Fig. 1) are shown by points. Time windows of equal width are set for input signals and noise. Fourier transforms of the signals and power spectral densities are derived using an FFT program in Fortran (code 12-4 in Ref.[16]).

\subsubsection{Gamma distribution as an approximation to the signal}

It seems that the reconstructed input signal of the Cherenkov radiation induced by EAS can be approximated by some kind of common functions.

We have tested three suitable distributions (normal "Gaussian", exponentially modified "exGaussian", and the Gamma distribution) as an analytic approximation to the input Cherenkov signal from the EAS event detected 21.10.2012. As a goodness of fit criterion is used the minimum sum of square deviations from experimental points (Fig. 3). The gamma distribution having the minimum sum is turned out to be the best approximation to the reconstructed Cherenkov signal.

For our analysis we employ a three-parameter family of probability distributions

$$
f_{\gamma}(t)=\frac{1}{\Gamma(\kappa) \theta^{\kappa}}\left(t-t_{0}\right)^{\kappa-1} e^{-\left(t-t_{0}\right) / \theta}
$$

where $t_{0}$ is an initial point such that: $f_{\gamma}=0$ at $t<t_{0} ; \kappa$ and $\theta$ are shape and scale parameters; and $\Gamma$ is normalizing gamma function. The mean value and variance are given by $\langle t\rangle=t_{0}+\kappa \theta, D=\kappa \theta^{2}$. 
Table 1: Duration of input signals, FWHM, ns.

\begin{tabular}{lrrrrrrrrrrrr}
\hline \hline Channel number & 1 & 2 & 3 & 4 & 5 & 6 & 7 & 8 & 9 & 10 & 11 & 12 \\
\hline Wiener deconvolution & 41.6 & 51.7 & 37.3 & 35.6 & 42.5 & 32.1 & 56.1 & 45.7 & 45.1 & 40.0 & 47.5 & 58.0 \\
Gamma distribution fit & 41.5 & 52.0 & 38.1 & 36.6 & 42.4 & 35.2 & 55.8 & 45.2 & 47.3 & 39.2 & 47.8 & 58.0 \\
A simple algorithm & 26.2 & 52.3 & 28.6 & 27.7 & 29.8 & 30.1 & 66.7 & 51.8 & 54.2 & 29.3 & 33.5 & 37.6 \\
Ratio (Wiener/Simple) & 1.59 & 0.99 & 1.31 & 1.28 & 1.43 & 1.07 & 0.84 & 0.88 & 0.83 & 1.36 & 1.42 & 1.54 \\
\hline \hline
\end{tabular}

Comparison of signal durations is given in the first two rows of Table 1. A difference in the full width at half-maximum (FWHM) of the deconvolved and fitted gamma distributions is less than $10 \%$.

\subsubsection{A toy model}

We introduce a simple model in this section in order to illustrate the reconstruction of the input signal and in order to elucidate the influence of noise on the Wiener deconvolution result. In accordance with the conclusion in the previous section, we use a gamma distribution as the input signal and the real impulse response given in Section 3.1.

A background of white noise (a sequence of uncorrelated signals) is modeled using a pseudorandom number generator in Fortran $x_{i}=2 \times A_{\text {noise }} \times(\operatorname{ran}(i)-0.5)$, where $A_{\text {noise }}$ is a constant noise amplitude: $x_{i} \in\left(-A_{\text {noise }}, A_{\text {noise }}\right)$. The noise is added to $f_{\text {out }}$, which is formed by the convolution $\left(f_{\text {in }} * g\right)$. The signal-to-noise ratio is defined by the variances of signal, $\sigma_{\text {signal }}^{2}$, and noise, $\sigma_{\text {noise }}^{2}$, expressed in decibels: $\mathrm{SNR}=10 \lg \left(\sigma_{\text {signal }}^{2} / \sigma_{\text {noise }}^{2}\right)$. The ratio is calculated in the same time window for signal and noise.

The input signal is reconstructed using Wiener deconvolution as described in Section 3.2.1. The resulting signal is compared with the true input in Fig. 4 for different SNR. Increasing the noise fraction leads to a distortion of the reconstructed signal. The necessary condition on SNR in order to have an artifact in the Wiener deconvolution less than $1 \%$ is SNR $>56 \mathrm{~dB}$.

\section{Light version of signal reconstruction with input gamma distribution}

Assuming that a gamma distribution approximates the input signal from EAS Cherenkov radiation, we have obtained a useful, simplified signal reconstruction algorithm without deconvolution. Namely, the representation of $f_{\text {in }}$ as

$$
f_{i n}^{\gamma}(t)=\frac{1}{\Gamma(\kappa) \theta^{\kappa}}\left(t-t_{0}\right)^{\kappa-1} e^{-\left(t-t_{0}\right) / \theta}
$$

merely leaves three parameters to be fitted to the output signal $f_{\text {out }}=\left(f_{\text {in }}^{\gamma} * g\right)$. For that, we adjust the time window to include the selected output signal, fit the free parameters of $f_{i n}^{\gamma}$ in order to obtain the measured Cherenkov signal, thus decreasing the noise.

It appears that by having three adaptable parameters of the input gamma distribution, we may obtain a sufficient description of the observed output signal. However, as was shown with the toy 

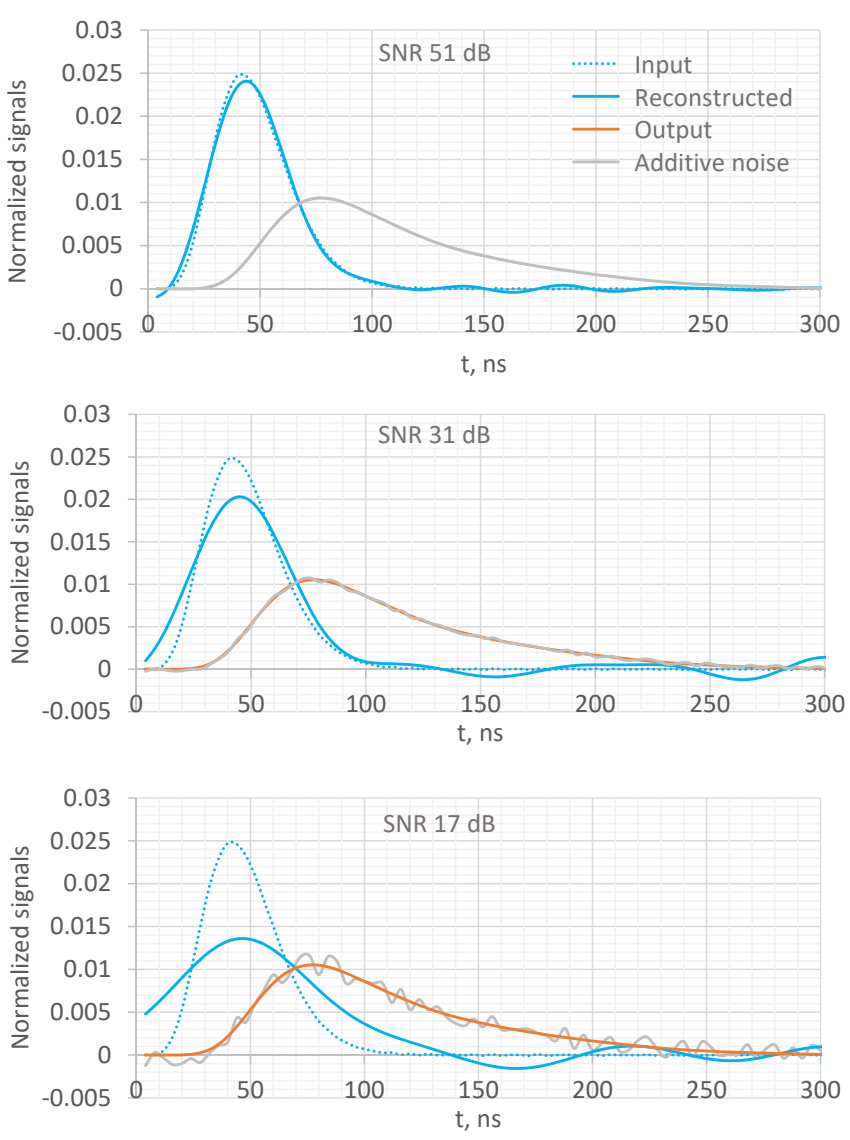

Figure 4: Wiener deconvolution of a noisy signal in a toy model.

model, the additive noise influence leads to a distortion of the deconvolved input signal, increasing with increased noise fraction, at least for the Wiener algorithm.

In order to estimate the accuracy of the reconstructed signal, two independent methods may be compared: a) Wiener deconvolution and b) a simple reconstruction of the input signal in the form of a gamma distribution. A comparison of durations of the input signals is presented in the final two rows of Table 1. The ratio of FWHM of Wiener deconvolution results and the gamma distribution width is, on average, $1.21 \pm 0.26$. This result may be attributed to $\mathrm{SNR}=(45 \pm 4) \mathrm{dB}^{1}$ inherent in measurements of the telescope of the Yakutsk array.

\section{Reconstruction of saturated signals}

Some portions of the detected signals in the experiments are saturated because of the maximum capacity of the instruments having been exceeded. This behavior is commonplace in cosmic ray physics, particularly in EAS studies in which there is a wide dynamic range of energy $\left(10^{14}, 10^{20}\right)$ $\mathrm{eV}$.

\footnotetext{
${ }^{1}$ Estimated as $\operatorname{SNR}=10 \lg \left(\left(\sigma_{\text {Fout }}^{2}-\sigma_{\text {noise }}^{2}\right) / \sigma_{\text {noise }}^{2}\right)$ in 12 channels
} 

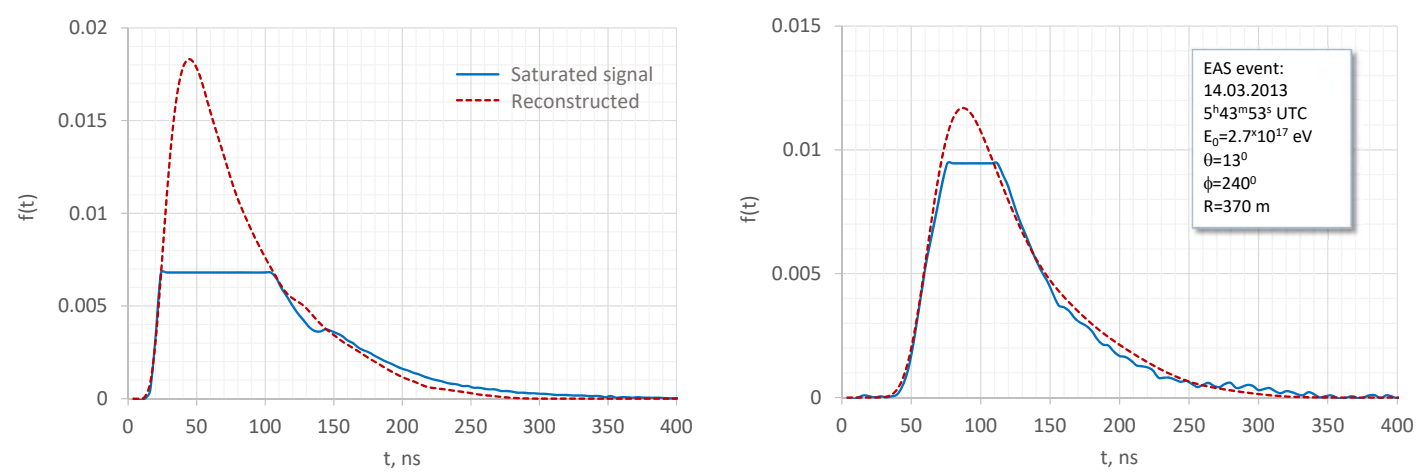

Figure 5: Reconstruction of two saturated output signals from EAS event denoted in the right panel.

While a conventional approach to repairing the saturated signal is to avoid it, in some cases, when the most physically interesting information is lost, or the measurement is unique, it is preferable to recover the signal in the primary saturation domain. Several methods for the reconstruction of saturated signals have been proposed previously (see for instance [17, 18]).

In our case, the input Cherenkov radiation signal can be approximated using a gamma distribution, offering a simple method for reconstruction of the saturated signal. The algorithm is the same as in the previous Section, besides ignoring the cut-off portion of the signal. Obviously, the accuracy of a recovery depends on the fraction of signal that was lost to saturation.

In Fig. 5, two signals reconstructed using this method are shown. For an EAS event detected 14.03.2013, $5^{h} 43^{m} 53^{s}$ UTC, signals for two of the 32 wires in the telescope are saturated. In the second case, a relatively insignificant portion of the signal is lost and the reconstruction is more reliable - at least, in terms of signal duration.

\section{Conclusions}

We have described the application of digital signal processing in order to reconstruct Cherenkov radiation signals, induced by EAS, from the data of a telescope working in co-operation with the surface scintillation counters of the Yakutsk array.

The transfer function of the system is evaluated using a dark current impulse of the multianode PMT. Using this transfer function and a Wiener deconvolution algorithm, the input signal is reconstructed. The influence of noise parameterized with the signal-to-noise ratio is estimated using a toy model. It is found that the SNR of the WFOV Cherenkov telescope should be more than $56 \mathrm{~dB}$ in order to accurately reconstruct the input signal.

It is demonstrated that the Cherenkov radiation signals from EAS can be approximated by a gamma distribution. Consequently, a simple method for reconstruction of such signals is proposed that does not require deconvolution. Moreover, the reconstruction method can be used to recover saturated signals.

\section{Acknowledgments}

We would like to thank the Yakutsk array group for data acquisition and analysis. This study 
is supported in part by the Presidium of RAS (program 3), the Siberian Branch of RAS (program II.16.2.3), and RFBR (project 16-29-13019).

\section{References}

[1] I.M. Frank and I.E. Tamm, Coherent radiation of fast moving electron in the medium, Dokl. Akad. Nauk SSSR 14 (1937) 107.

[2] N. Budnev et al., TAIGA experiment: present status and perspectives, J. Inst. 12 (2017) C08018.

[3] W. Galbraith and J. Jelley, Light pulses from the night sky associated with CRs, Nature 171 (1953) 349.

[4] N.M. Nesterova and A.E. Chudakov, On the observation of Cherenkov radiation accompanying broad atmospheric showers of CRs, JETP 28 (1955) 384.

[5] M.N. Dyakonov et al., The use of Cherenkov detectors at the Yakutsk CR EAS array, Nucl. Instrum. Meth. A 248 (1986) 224.

[6] A.A. Ivanov, S.P. Knurenko, I.E. Sleptsov, Determining the primary CR energy from the total flux of Cherenkov light measured at the Yakutsk array, JETP 104 (2007) 872.

[7] R.T. Hammond et al., Cherenkov radiation in large CR air showers, IL Nuovo Cimento 1C (1987) 315.

[8] J.W. Fowler et al., A measurement of CR spectrum and composition at the knee, Astropart. Phys. 15 (2001) 49.

[9] A.A. Ivanov et al., Enhancement of the Yakutsk array by atmospheric Cherenkov telescopes to study CRs in the energy range above $10^{15} \mathrm{eV}$, ASTRA 6 (2010) 53.

[10] A.A. Ivanov et al., Wide field-of-view Cherenkov telescope for the detection of cosmic rays in coincidence with the Yakutsk extensive air shower array, Nucl. Instrum. Meth. A 772 (2015) 34.

[11] A.A. Ivanov and L.V. Timofeev, Temporal signatures of the Cherenkov light induced by extensive air showers of cosmic rays detected with the Yakutsk array, Int. J. Mod. Phys. D 20 (2011) 1539.

[12] M. Nakhostin, Zs. Podolyak, P.H. Regan Digital processing of signals from $\mathrm{LaBr}_{3}:$ Ce scintillation detectors, J. Inst. 9 (2014) C12049.

[13] V.A. Kolosov, Thesis, Lebedev Physical Institute, Moscow 2001.

[14] J.W. Cooley and J.W. Tukey, An algorithm for the machine calculation of complex Fourier series, Math. of Comput. 19 (1965) 297.

[15] N. Wiener, Extrapolation, Interpolation, and Smoothing of Stationary Time Series, Cambridge, Mass: MIT Press. 1964.

[16] S.W. Smith, The Scientist \& Engineer's Guide to Digital Signal Processing, California Technical Publishing, San Diego 1999.

[17] W. Yang and Y. Ben-Zion, An algorithm for detecting clipped waveforms and suggested correction procedures, Seism. Res. Lett. 81 (2010) 53.

[18] Y. Liu et al., Recovery of Saturated $\gamma$ Signal Waveforms by Artificial Neural Networks, arXiv:1810.08200, (submitted to Nucl. Instrum. Meth. A). 